\title{
Intradermal fillers for minimally invasive treatment of facial aging
}

\section{Zhi Yuan (William) Lin', Vishva Shah'1, Arvind Dhinakar ${ }^{1}$, Lara Yildirimer², Wen-Guo $\mathrm{Cui}^{3}$, Xin Zhao ${ }^{1}$}

${ }^{1}$ The Key Laboratory of Biomedical Information Engineering of Ministry of Education, Xi'an Jiaotong University, School of Life Science and Technology, Xi'an 710049, Shaanxi, China.

${ }^{2}$ Centre for Nanotechnology and Regenerative Medicine, UCL Division of Surgery and Interventional Science, University College London, London WC1E 6AU, UK.

${ }^{3}$ Department of Orthopedics, The First Affiliated Hospital of Soochow University, Orthopedic Institute of Soochow University, Suzhou 215006, Jiangsu, China.

Address for correspondence: Dr. Xin Zhao, The Key Laboratory of Biomedical Information Engineering of Ministry of Education, Xi'an Jiaotong University, School of Life Science and Technology, Xi' an 710049, Shaanxi, China. E-mail: xinzhao6@xjtu.edu.cn

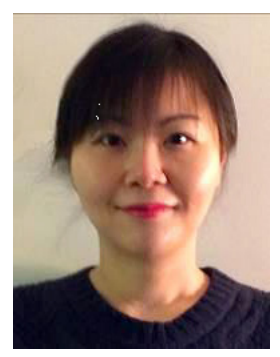

Xin Zhao is an associate professor at Xi'an Jiaotong University, China. Prior to starting this appointment, she was a postdoctoral fellow at Medical School and School of Engineering and Applied Sciences at Harvard University. Her research involves engineering the micro- and nanoscale systems for tissue regeneration and drug delivery.

\begin{abstract}
The ever-increasing interest in retaining a youthful physical appearance has facilitated the development of various minimally invasive dermatological techniques. The use of intradermal fillers can be incorporated into dermatological practices with minimal overhead costs. This strategy addresses facial volume loss and dynamic lines, which are the main features of facial aging. Moreover, intradermal fillers provide an array of flexible treatment options for a balanced and holistic result to dermatological practitioners. This paper reviews the different intradermal fillers categorized by biodegradable and non-permanent fillers including collagen based materials, hyaluronic acid and autologous fat, semi-permanent fillers including poly methyl methacrylate, poly-L-lactic acid and calcium hydroxyapatite microspheres, and permanent fillers including silicone. A discussion is provided of the commercial products made of these materials and their clinical efficacy in the treatment of facial aging.
\end{abstract}

Key words:

Intradermal fillers; facial aging; volumization; biodegradable and non-permanent fillers; semipermanent fillers; permanent fillers

\begin{tabular}{|l|l|}
\hline \multicolumn{2}{|c|}{ Access this article online } \\
\hline Quick Response Code: & Website: \\
\hline & http://parjournal.net \\
\cline { 2 - 3 } & \\
\hline
\end{tabular}

This is an open access article distributed under the terms of the Creative Commons Attribution-NonCommercial-ShareAlike 3.0 License, which allows others to remix, tweak and build upon the work non-commercially, as long as the author is credited and the new creations are licensed under the identical terms.

For reprints contact: service@oaepublish.com

How to cite this article: Lin ZY, Shah V, Dhinakar A, Yildirimer L, Cui WG, Zhao X. Intradermal fillers for minimally invasive treatment of facial aging. Plast Aesthet Res 2016;3:72-82.

Received: 05-11-2015; Accepted: 25-11-2015 


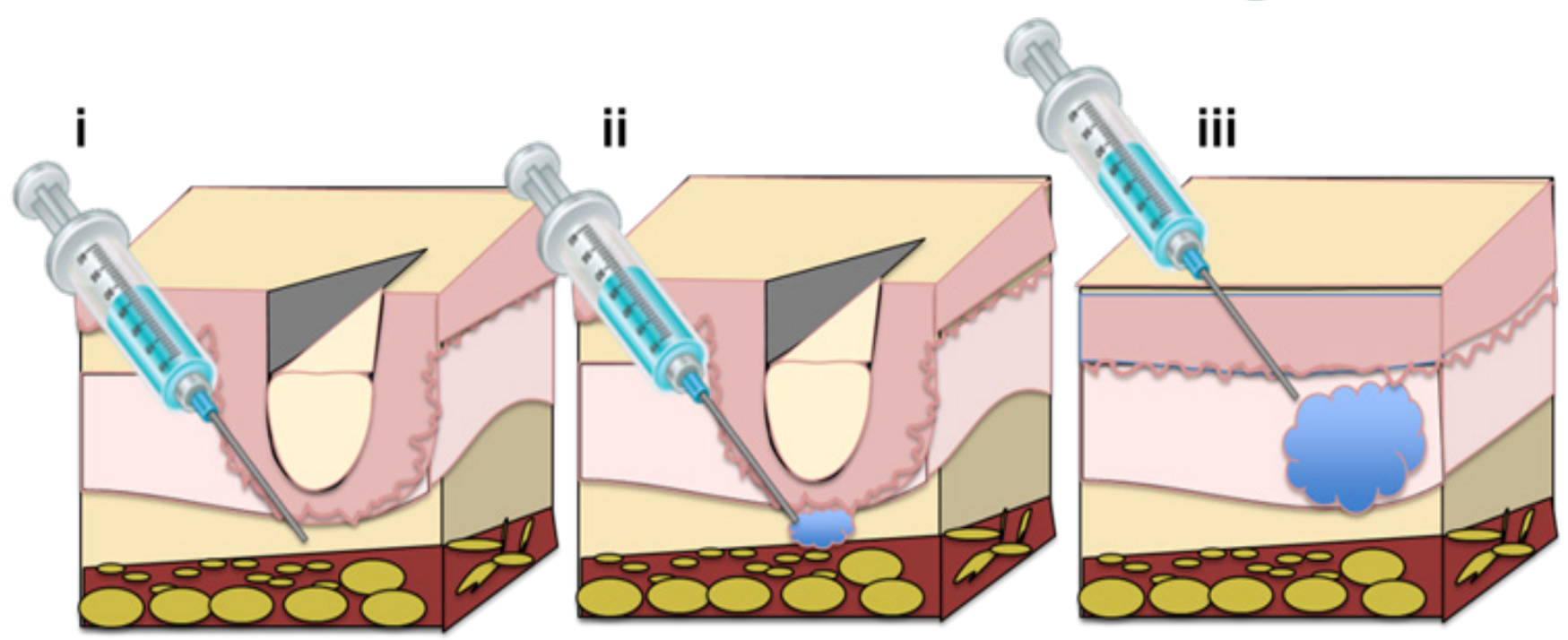

Figure 1: Procedure protocol for injection of intradermal fillers for the treatment of facial aging. (i) The depth of needle insertion ranges from superficial to mid-dermis and is dependent on both wrinkle type and filler type; (ii) release of intradermal fillers through the needle; (iii) filler insertion results in smoothed wrinkles and the effect is long-lasting ${ }^{|31|}$

\section{INTRODUCTION}

A youthful face is characterized by fullness, balance, smoothness, and the absence of facial lines or wrinkles. During the aging process, the outer shell of the human body changes its appearance. These skin alterations are often narrowly focused on facial wrinkles, one of the most visible signs of the aging process. Therefore, the majority of approaches intending to reverse skin-aging signs target the treatment of wrinkles. ${ }^{[1]}$ However, it has recently become more evident that the treatment of wrinkles alone is not sufficient to restore a youthful facial appearance. As aging continues, the subcutaneous fat pads shrink, leading to the loss of structural support which in turn creates sagging of the overlying skin. The loss of facial volume beneath the skin (e.g. subcutaneous fat, muscle) is thus considered to be the major contributor to the appearance of advanced age. ${ }^{[1]}$

A number of minimally invasive dermatological techniques have been developed for facial rejuvenation. Injectable dermal fillers can be injected through a needle into the upper layers of the dermis to treat superficial fine wrinkles or injected into the deep dermis or subcutaneous space for facial volume augmentation ${ }^{[2]}$ [Figure 1]. Dermal fillers are deposited in a slow and steady manner and fill central folds, resulting in a natural, long-lasting outcome. Crease depth, desired outcome, and the patient's financial situation are factors which need to be taken into account when deciding the amount of product to be used.

This paper focuses on intradermal fillers, chosen due to their ability to alleviate aging-related conditions and their ability to be easily incorporated into the armamentarium of outpatient cosmetic procedures with minimal overhead costs.

\section{TYPES OF INTRADERMAL FILLERS}

Intradermal fillers can be roughly divided into three categories depending on their durability: (1) biodegradable and non-permanent agents [e.g. bovine collagen, porcine collagen, human collagen, hyaluronic acid (HA) and autologous fat]; (2) semi-permanent agents [e.g. poly methyl methacrylate (PMMA) microspheres, poly-L-lactic acid (PLA) and calcium hydroxyapatite (CaHA)]; (3) permanent agents (e.g. silicone). The commercial products, their materials, and their clinical efficacy are summarized in Table 1.

\section{Biodegradable and non-permanent fillers Bovine collagen}

Bovine collagen has been in use for over 20 years as intradermal filler. It is extracted from bovine tendon, dermis and bone. ${ }^{[3]}$ Once injected it has the ability to form a rigid structure composed of fibrils, with an axial periodicity of native collagen which stays intact to help correct facial defects. ${ }^{[4]}$ Since its approval by the Food and Drug Administration (FDA) in 1981, highly purified bovine collagen products have been used as fillers in more than 2 million patients. ${ }^{[5]}$ Different forms of bovine collagen are currently available, including Zyderm ${ }^{\circledR}$ I and II (Inamed Aesthetics, Santa Barbara, CA, USA), composed of $3.5 \%$ or $6.5 \%$ bovine dermal collagen suspended in a phosphate buffer solution, respectively. Both are used for the correction of superficial lines including scars, peri-orbital lines and crows's feet. ${ }^{[6]}$ Similarly, Zyplast ${ }^{\circledR}$ (Inamed Aesthetics), is composed of $3.5 \%$ collagen with the addition of $0.0075 \%$ glutaraldehyde, which helps to strengthen the collagen fibers and prolong the duration of action. ${ }^{\left[{ }^{6}\right]}$ Zyplast ${ }^{\circledR}$ is primarily used to treat deeper scar tissue including acne scars and the vermillion border of the lip..$^{\mid 6]}$

Various distinctive studies have demonstrated the efficacy of Zyderm ${ }^{\circledR}$ in the cosmetic industry including a study conducted by Nicolle, ${ }^{, 7]}$ which showed the effectiveness of Zyderm ${ }^{\circledR}$ II in 350 patients treated for various facial contour defects including acne, glabellar frown lines, periorbital lines and naso-labial folds. Eighty percent of the patients treated were satisfied with their results although 5 patients showed signs of a positive localized reaction to the test dosage of Zyderm ${ }^{\circledR}$ II. ${ }^{[7]}$ Additionally, a clinical 
Table 1. Commercially available facial fillers

\section{Filler \\ Biodegradable and non-permanent fillers}

Bovine collagen $(Z y d e r m \AA$ Extracted from bovine I and II, Zyplast®)

Porcine collagen

(Evolence $^{\mathrm{TM}}$, TheraFill囚)

Human collagen

(Cosmoderm®)

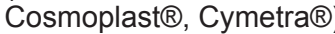

HA (Restylane $®$,

Juvederm $30^{\mathrm{TM}}$, Juvederm

Ultra $^{\mathrm{TM}}$, Juvederm

Ultra Plus ${ }^{\mathrm{TM}}$,Perlane $\AA$

Hylaform $®$ Hylaform Plus $®$ )

Autologous fat

(Radiesse®)

\section{Semi-permanent fillers}

PMMA microspheres

(Arteplast $\circledast$, Artecoll $\circledast$,

Artefill $\circledast$, Dermalive $®$ )

Non-biodegradable,

\section{Function} tendons, dermis and bones; injected to correct facial defects

Extracted from porcine tendons, dermis and bones; injected to fill wrinkles

Derived from human dermis to treat wrinkles

and scars

Very commonly found

substance; usually derive from either bacteria or rooster combs

Used for soft tissue augmentation
Uses

Forms a rigid structure composed of fibrils, with an axial periodicity of native collagen that stays intact

\section{Volume enhancer} (nasolabial)

FDA-approved for deep scars, superficial and deep wrinkles

Volume and contouring
Pros

Cons

usually found to last between 4-6 months for

Can cause adverse immune reactions

rhytids and 6-9 months for scars regardless of which product is used

Less immunogenic than bovine collagen

May elicit immunogenic reaction, although it may not be considered adverse

Skin testing is not required May cause adverse before use reactions

Skin testing is not required Usually associated with before use short-lived side effects
Facial volume augmentation and wrinkle reduction

Can use large volume without immunoreaction. biocompatible, synthetic polymer

Can be easily fabricated to 30 to $40 \mu \mathrm{m}$ in diameter, small enough
PLA (Sculptra/Fill囚)

CaHA (Radiesse®)
Biodegradable; usually derived from renewable sources; injected into either the deep dermis or subcutaneous layer.
Volume and contouring (nasolabial, radial upper lip lines, glabella lines, corner of mouth lines)
Restoration of facial fat loss (nasolabial folds, labiomental creases, lip, etc.) marionette lines, upper to pass through the needles but big enough to avoid phagocytosis; inexpensive, readily accessible and simple to apply

Assists with volume restoration while simultaneously increasing dermal thickness in the face; reduced safety concerns; very good risk-benefit profile for HIV patients and for cosmetic purposes

Correction of moderate-to- Biocompatible; skin severe wrinkles and folds; testing is not required volumization of facial soft- before use tissue;correction of the signs of facial lipoatrophy

Volume and contouring;injected into the deep dermis or subdermal plane
Low maintenance and cost; permanent
Fat needs to be first extracted from another source and grafted fat may be inconsistent quality.

Some immediate adverse reactions mainly associated with the development of lumps or nodules

Volumization disappears within a few months; dermal fibroplasia; Results not immediate (more than 2 years)

Side effects more common; may be unsafe to use for glabellar or periorbital lines

Adverse complications; cannot be removed after implantation

FDA: Food and Drug Administration; HA: hyaluronic acid; PMMA: poly(methyl methacrylate); PLA: poly-L-lactic acid; CaHA: calcium hydroxyapatite

study by Bailin and Bailin ${ }^{[8]}$ in which 8 patients were treated with Zyplast ${ }^{\circledR}$ and Zyderm ${ }^{\circledR}$ I and II for surgical scars, acne scars and rhytids or a combination of these found that both products were effective. Results lasted between 4-6 months for rhytids and 6-9 months for scars regardless of which product was used. ${ }^{[8]}$

Skin testing is essential for patients undergoing treatment with bovine collagen because it is extracted from an animal source and may provoke a reaction. Generally, two skin tests spaced 2 or 4 weeks apart are recommended. The skin test is performed by intradermal injection of $0.1 \mathrm{~mL}$ of collagen onto the volar forearm to detect any pre-existing allergy to Zyderm ${ }^{\circledR}$ or its counterparts. ${ }^{[9]}$ It is suggested that the occurrence of local sensitive reaction ranges from $3 \%$ to $5 \%$. Various clinical tests and studies have shown that Zyderm ${ }^{\circledR}$ and Zyplast ${ }^{\circledR}$, if not used effectively, can cause adverse reactions. ${ }^{[9]}$ These reactions are often classified as immune responses to the foreign material being injected into the body. ${ }^{[9]}$ In general, Zyplast ${ }^{\circledR}$ and Zyderm ${ }^{\circledR}$ may elicit adverse reactions such as hypersensitivity, herpes virus reactivation, bacterial infection, bruising, and local necrosis. Therefore, it is crucial to follow strict procedures regarding the skin testing protocol, as well as to compare it to other fillers to determine which one if appropriate for use.

Porcine collagen

Porcine collagen is extracted from porcine tendon, dermis 
and bone. In recent years, porcine collagen has been marketed as an intradermal filler under the brand name of Evolence $^{\mathrm{TM}}$ (ColBar LifeScience Ltd., Herzliya, Israel). Studies involving human medical devices have shown that porcine collagen is less immunogenic than bovine collagen, although the amount of published data regarding its potential as intradermal filler is still relatively insignificant. ${ }^{[10]}$ In a phase I clinical trial with 12 patients receiving treatment for nasolabial folds, both Evolence ${ }^{\mathrm{TM}}$ and Zyplast ${ }^{\circledR}$ were found to be safe and effective. ${ }^{[10]}$ The degree of improvement in wrinkles, graded by blinded examiners, was observed to be much greater for Evolence ${ }^{\mathrm{TM}}$ at both the 6-month and 18-month visiting periods. ${ }^{[11]}$ A larger randomized, doubleblinded trial found no significant difference in effectiveness between Evolence ${ }^{\mathrm{TM}}$ and NASHA (Restylane ${ }^{\circledR}$ ) in the treatment of nasolabial folds at the 6-month assessment. Although there were no positive skin tests against porcine collagen, $6.1 \%$ of patients developed $\operatorname{IgG}$ antibodies to porcine collagen. Although there were immunogenic reactions, it is important to note that none of them were considered to be adverse in nature. ${ }^{[12]}$

Another study was conducted comparing the safety and efficacy of TheraFill ${ }^{\circledR}$, a porcine collagen filler, to a bovine collagen filler for the treatment of nasolabial folds. In total 61 patients were evaluated in this randomized, double-blinded and split-face study over a 12-month period. The Wrinkle Severity Rating Scale (WSRS) rating of TheraFill ${ }^{\circledR}$ was shown to be higher than that of bovine collagen filler by a small margin, although not enough to be considered statistically significant. Both fillers were similarly effective and safe without eliciting severe adverse reactions, suggesting that TheraFill ${ }^{\circledR}$ could suitably replace bovine collagen filler. ${ }^{[13]}$

Many studies have been performed which assess the efficacy of bovine and porcine dermal fillers. The aforementioned studies discuss the adverse/allergic responses and the efficacy of treatment provided by these fillers. ${ }^{[14,15]}$ More comparative studies have been done between bovine or porcine and other fillers to choose the safest and most preferred treatment. ${ }^{[16]}$ With the advent of new technology, the cosmetic industry has moved on to various other types of intradermal fillers including human collagen, smooth gel hyaluronic acid, poly-L-lactic acid (PLA), poly(methyl methacrylate) (PMMA) microspheres, etc., which have proven to be better alternatives to bovine and porcine fillers. These are discussed in detail below.

\section{Human collagen}

Collagen has traditionally been extracted from animal sources or cadavers. Recently researchers have proposed obtaining human collagen from other sources including adipose tissue obtained after liposuction ${ }^{[17]}$ and from yeast or bacteria used for the production of recombinant human collagen. ${ }^{[18]}$ Moreover, human collagen can now also be synthesized in the lab as discovered by Raines. ${ }^{[19]}$ Cosmoderm ${ }^{\circledR}$ and Cosmoplast ${ }^{\circledR}$ (Inamed Aesthetics) are some of the currently available products made of human collagen; both contain lidocaine and have received FDA approval. The collagen content is produced from a single human dermal fibroblast cell line. Cross-linking between lysine residues on collagen and glutaraldehyde makes Cosmoplast ${ }^{\circledR}$ less prone to degradation. Cosmoplast ${ }^{\circledR}$ is used for the treatment of deeper wrinkles and deep scars whereas Cosmoderm ${ }^{\circledR}$ is used for the treatment of superficial wrinkles, both with effects lasting for 3-7 months. ${ }^{[20]}$ The Inamed clinical study of human collagen immunogenicity concluded with a 95\% upper confidence interval that the probability of a hypersensitivity reaction from either Cosmoplast ${ }^{\circledR}$ or Cosmoderm ${ }^{\circledR}$ is less than $1.3 \% .^{[21]}$

AlloDerm is a sheet derived from human dermis that requires a multi-step treatment to ensure optimal efficacy. Cymetra ${ }^{\circledR}$ (LifeCell Corp., Branchburg, NJ, U.S.A.) is an injectable form of AlloDerm. ${ }^{[22]}$ Very few trials have been conducted comparing human-derived collagen with other types of fillers. A randomized trial comparing Zyplast ${ }^{\circledR}$ (bovine collagen) and Cymetra ${ }^{\circledR}$ (human collagen) injections to the upper lip showed that Cymetra ${ }^{\circledR}$ had a much higher posttreatment value than Zyplast ${ }^{\circledR}$ by the 12 -month visit, with bruising being the most commonly observed adverse effect for both fillers. ${ }^{[23]}$ Because Cymetra ${ }^{\circledR}$ is of human origin, skin testing is not required prior to use.

Although collagen treatment is beneficial for most individuals, it has its limitations. Patients who are sensitive to bovine collagen must instead use human collagen, which is typically more expensive. Individuals with autoimmune diseases should completely avoid the use of foreign collagen. Finally, the results typically last for a short period of time as the body absorbs external collagen naturally.

\section{Hyaluronic acid}

HA is a very commonly found substance which is usually derived from either bacteria or rooster combs for use as an intradermal filler. ${ }^{[24]}$ Although several products containing HA-related proteins have shown a theoretical risk of hypersensitivity, there is no requirement for skin testing prior to use. ${ }^{[24]}$

Restylane ${ }^{\circledR}$, a type of non-animal stabilized hyaluronic acid (NASHA), is a stable, partially cross-linked form of HA. It is fermented by bacteria and has a long track record of safety (over 10 years) as an intradermal filler. Due to rapid degradation of hyaluronic acid in the skin, cross-linking is required in order to produce a hydrophilic HA polymer. Because no anesthetic is associated with Restylane ${ }^{\circledR}$, topical anesthesia or a nerve block may be administered prior to its use. Narins et al. ${ }^{[25]}$ conducted a double-blinded, randomized, and longitudinal comparison study between Restylane ${ }^{\circledR}$ and Zyplast ${ }^{\circledR}$ for treatment of the nasolabial fold. In the study involving 138 patients, the Wrinkle Severity Rating Scale (WSRS) (with 1 being the lowest and 5 being the most severe) and Global Aesthetic Improvement Scale (GAIS) (on a scale of 5 ranging from "worse" to "very much improved") were used at 2, 4, and 6 months after baseline. Both groups showed similar improvements with $90 \%$ of patients exhibiting improvement by 1 or 2 scores according to the WSRS rating. At 6 months, more patients showed improvement with Restylane ${ }^{\circledR}$ ( $56.9 \%$ for WSRS; $62 \%$ for GAIS) than with Zyplast ${ }^{\circledR}$ ( $9.5 \%$ for WSRS; $8 \%$ for GAIS). Reactions (e.g. swelling, tenderness, bruising, redness and 


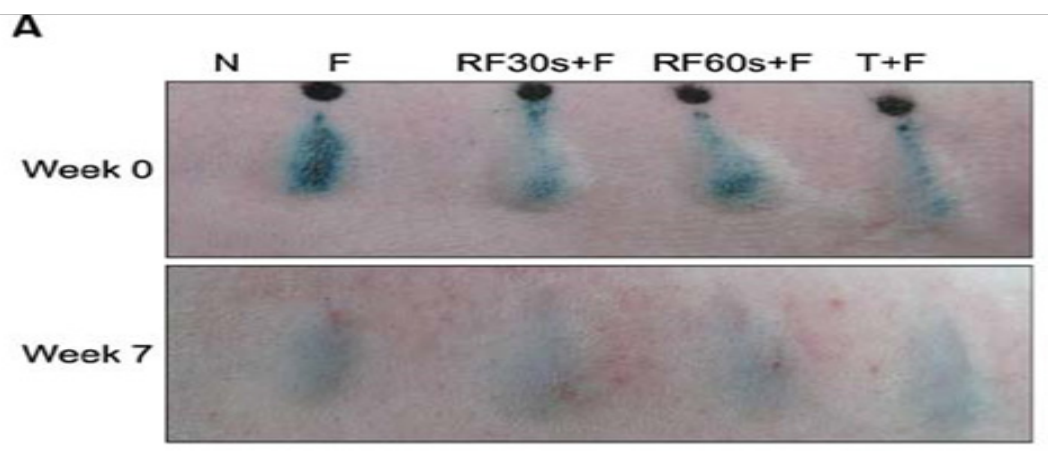

B

Control
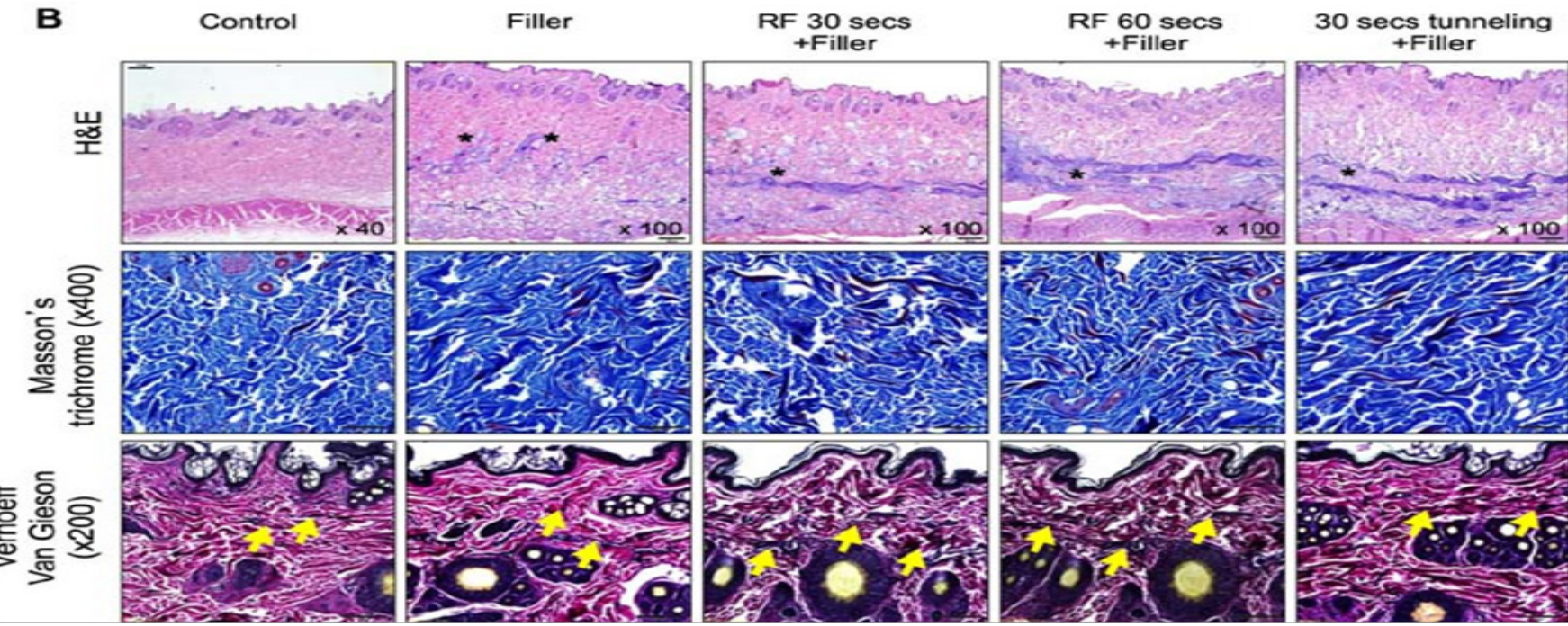

Figure 2: (A) Filler injections after radiofrequency (RF) treatment results in volumization. N: normal; F: Glytone 3 filler injection; RF30s+F: RF treatment for $30 \mathrm{~s}$, RF60s +F: RF treatment for $60 \mathrm{~s}$, both before filler injections through tunneling method; T+F: filler injected after tunneling without RF treatment for $30 \mathrm{~s}$. (B) “*” sign indicates areas injected with fillers. Extracellular matrix components stained with Masson's trichrome show the treatment with both filler and RF enhances collagen bundle deposition (blue) and fibroplasia (red) Dermis stained with Verhoeff-Van Gieson shows that RF treatment resulted in shorter elastin fibers ("arrow" sign). Image used with permission. ${ }^{[3}$

pain) were observed in $93.5 \%$ of Restylane ${ }^{\circledR}$-treated sites and $90.6 \%$ of Zyplast ${ }^{\circledR}$-treated sites, and lasted for 7 days at most. Reactions were also observed after 14 days or more in 12 patients, with 10 reactions in Restylane ${ }^{\circledR}$-treated sites and 11 reactions in Zyplast ${ }^{\circledR}$-treated sites. Local erythema was the most common delayed reaction. All reactions spontaneously subsided without treatment and were not considered to be hypersensitivity reactions. A similar study was conducted comparing three formulations of HA filler (Juvederm $30^{\mathrm{TM}}$, Juvederm Ultra ${ }^{\mathrm{TM}}$ and Juvederm Ultra Plus ${ }^{\mathrm{TM}}$; Allergan) with Zyplast ${ }^{\circledR}$ for the treatment of nasolabial folds. This study also concluded that the effect of HA fillers was much longer-lasting than that of bovine collagen. ${ }^{[16]}$

A Scandinavian double-blinded, randomized, longitudinal comparison study with 68 patients was conducted between Perlane ${ }^{\circledR}$ (a form of NASHA produced by Medicis Aesthetics, Scottsdale, AZ, USA) and Zyplast ${ }^{\circledR}$ for the treatment of nasolabial folds. ${ }^{[26]}$ Using the GAIS rating, both showed "much or very much improved" effects $\left(89.7 \%\right.$ for Perlane ${ }^{\circledR}$ and $86.8 \%$ for Zyplast ${ }^{\circledR}$ ) at the baseline period ( 2 weeks after injection). Perlane ${ }^{\circledR}$ consistently had higher WSRS ratings during the 9-month follow-up period. Reactions were found at the injection sites for both $\left(17.6 \%\right.$ for Perlane ${ }^{\circledR}$ and $30.9 \%$ for Zyplast $\left.{ }^{\circledR}\right)$. Three patients showed delayed reactions but none were considered to be hypersensitivity reactions.

A recent study regarding the use of NASHA showed that it stimulates the production of new collagen in human skin. In this study, each of 11 volunteers received 3 filler injections into one forearm and 3 isotonic saline vehicle injections into the contralateral forearm. Tissue biopsies results taken 4 and 13 weeks after injection revealed increased collagen deposition around the filler, higher type I procollagen marker staining, upregulation of type I and II procollagen expression, and elongated fibroblast morphology at the injection site. Various mechanisms have been proposed in an attempt to elucidate the de novo collagen production, including mechanical fibroblast stretching, growth factor stimulation, and inhibition of collagen degradation. Inhibition of collagen degradation was proposed after observing the expression of matrix metalloproteinases and their regulators in skin treated using NASHA. ${ }^{[27]}$

Double-blinded, randomized studies have also been conducted with Hylaform ${ }^{\circledR}$ (Inamed Aesthetics), an FDAapproved $H A$ that is extracted from rooster combs. Hylaform ${ }^{\circledR}$ had similar efficacy compared to Zyplast ${ }^{\circledR}$ for up to 4 months in 300 patients treated for nasolabial folds, with few adverse effects and no allergic reactions. ${ }^{[28]} \mathrm{A}$ small randomized longitudinal study was also conducted with Hylaform Plus ${ }^{\circledR}$, which contains bigger cross-linked molecules with a larger average particle size than its Hylaform ${ }^{\circledR}$ counterpart. The study was conducted in comparison to Restylane $\AA$ for the treatment of nasolabial folds and examiners were blinded during evaluation. At 3, 4.5, and 6 months, Restylane ${ }^{\circledR}$ 
was found to have a significantly lower WSRS rating than Hylaform Plus ${ }^{\circledR} .^{[29]}$

A randomized, split-face study was conducted for 12 months to assess the efficacy and safety of three HA formulations: HA-1 (Belotero Basic/Balance), HA-2 (Restylane), and HA-3 (Juvéderm Ultra 3/Juvéderm Ultra Plus XC) in the treatment of nasolabial folds. ${ }^{[30]}$ Participants were randomly assigned to one of two study groups, each with twenty participants. Each participant in group 1 received HA-1 on one side and HA-2 on the other, whereas participants in group 2 received HA-1 on one side and HA-3 on the other. Fillers were administered at the baseline visit, which took place during the initial phase, with follow-up injections at months $1,6,9$, and 12. All three of the HA formulations showed minimal adverse reactions, the most common of which was erythema. The mean pre-treatment and post-treatment nasolabial fold severity ratings did not show statistically significant differences between the groups. ${ }^{[30]}$

$\mathrm{HA}$ is the most widely used filler yet has a relatively short duration of action, resulting in the need for constant maintenance with frequent injections for optimal treatment. ${ }^{[31]}$ Recently, HA has been combined with radiofrequency $(\mathrm{RF})$ devices to prolong the duration of action of $\mathrm{HA}$, reducing the need for maintenance injections. ${ }^{[31,32]} \mathrm{RF}$ treatment has been found to play a crucial role in collagen remodeling, skin tightening, and collagen deposition, but has a limited capacity to restore lost volume. ${ }^{\mid 32]}$ Combination therapy in which RF is used immediately prior to treatment with a HA can compensate for both products' limitations while providing better treatment. Combination therapy was evaluated in both animal and human clinical studies where RF was delivered through an intradermal needle to creat tunnel-like setting inside the dermis and hypodermis. This theoretically acts as a barrier to external oxygen radicals and contains the HA filler, restricting its spread. ${ }^{[31]}$ The results of the animal study showed that RF treatment could increase procollagen production with time. The total volume was substantially increased with the RF treatment as compared to HA filler injections alone [Figure 2]. Specifically, in the areas injected, small filler particles were observed in the dermis. At sites injected with fillers following RF treatment, linear continuous filler distribution was observed in the mid-dermis, and in the lower dermis of sites treated with the tunneling method [Figure 2B]. The ECM components and dermis were stained with Masson's trichrome and VerhoeffVan Gieson, respectively. Enhancement of collagen bundle deposition and fibroplasia was observed with Masson's trichrome while the formation of short elastin fibers was observed with Verhoeff-Van Gieson staining [Figure 2B]. It is thus believed that the combination therapy of HA filler injection with RF is a biocompatible and long-lasting improvement in skin rejuvenation.

Although HA has shown excellent biocompatibility and efficacy in facial volumization, it has side effects including erythema, bruising, induration and edema. More importantly, they are all short-lived and therefore are of limited use in patients looking for longer lasting solutions.

\section{Autologous fat}

For more than a century, autologous fat transfer has been utilized for soft tissue secondary to its advantages including the absence of immune reaction and the feasibility in using large volumes. However, it suffers from various drawbacks including prior fat extraction from another source, and the inconsistent quality of fat due to the extraction technique.

A retrospective clinical study conducted by Kanchwala et al. . $^{[33]}$ examined facial volume augmentation and wrinkle reduction in 976 patients who received autologous fat, Restylane $\AA$, Hylaform ${ }^{\circledR}$ or Radiesse ${ }^{\circledR}$. Patients were followed at least 1 year from the time of injection for an assessment of infection rate, revision rate, and overall longevity. In this study, 378 patients had theirnasolabial folds treated with autologous fat. Self-limited bruising and swelling were observed 2-3 days after fat injection. Ten percent of patients required a second treatment 6-12 months following the first treatment, and the overall longevity was shown to be greater than 12 months. Forty-one and twenty-six patients received treatment of their nasolabial folds with Restylane ${ }^{\circledR}$ and Hylaform ${ }^{\circledR}$, respectively. Although only minimal complications were observed, they lasted for, on average, 4.5 and 3 months for Restylane ${ }^{\circledR}$ and Hylaform ${ }^{\circledR}$, respectively. One hundred and two patients were treated with Radiesse ${ }^{\circledR}$ for their nasolabial folds, and mild-to-moderate swelling was found at the injection sites for $24 \mathrm{~h}$ maximum. Twenty percent of patients required a second treatment at the same injection site less than 3 months following the first injection, and longevity was 11 months on average. Significant swelling and bruising that lasted for 1-2 weeks were noted in 87 patients who received autologous fat for lip augmentation, with nearly $30 \%$ of them requiring a second injection within 6 months secondary to variable resorption. Overall longevity was greater than 12 months. Twenty-four patients received Restylane ${ }^{\circledR}$ for lip augmentation with effects lasting for 4.5 months, while 17 patients received Hylaform ${ }^{\circledR}$ with 3-month longevity and slightly more swelling for 1-3 days. Twenty percent of patients required a second injection 3 months following the first injection. ${ }^{[33]}$

Biodegradable and non-permanent fillers last only for a few months, which is suboptimal in cases where maintenance and cost are issues. This problem gave rise to the advent of semi-permanent fillers as discussed in the next section.

\section{Semi-permanent fillers}

\section{Polymethyl methacrylate microspheres}

PMMA is a non-biodegradable, biocompatible, synthetic polymer used in various medical devices. Bovine collagen injections on the human face have been found to last only for 3 to 6 months, giving rise to the use of PMMA microspheres as intradermal fillers. ${ }^{[34]}$ The main advantage of microspheres is their size of 30-40 $\mu \mathrm{m}$ in diameter, which is small enough to pass through a needle but still large enough to avoid phagocytosis. $^{[35]}$

Arteplast ${ }^{\circledR}$, the first mixture which contained 20\% PMMA and $80 \%$ bovine collagen, was noted to give rise to foreign body reactions due to the presence of a number of microspheres less than $20 \mu \mathrm{m}$ which made them prone to phagocytosis. ${ }^{[35]}$ 

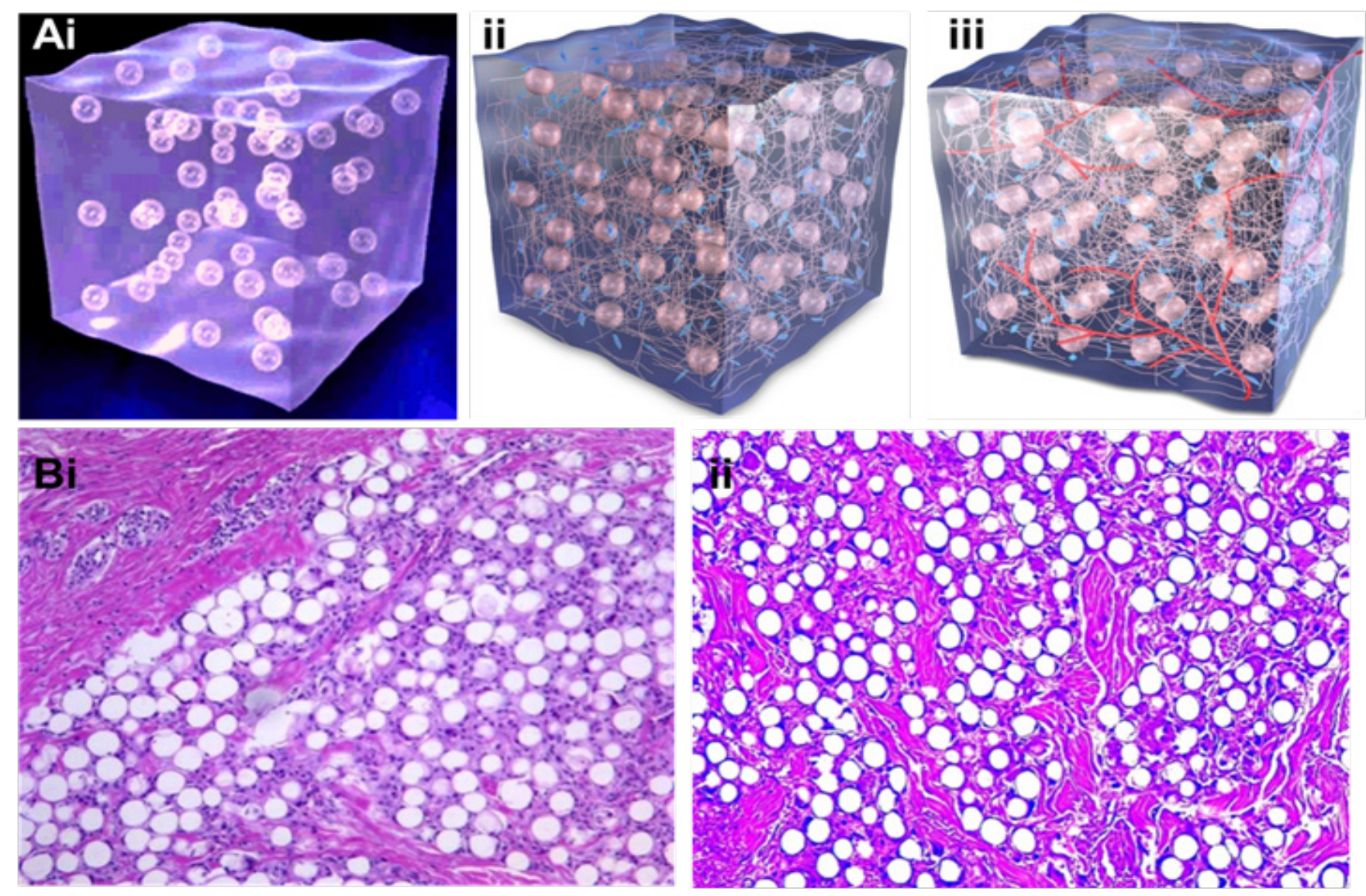

Figure 3: (Ai) ArteFill ${ }^{\circledR}$ composed of $20 \%$ PMMA microsphere $(30-50 \mu \mathrm{m})$ and $80 \%$ denatured bovine collagen; (Aii) tissue growth stimulated due to collagen maintaining the viscosity of microspheres; (Aiii) the presence of blood vessels is observed and bovine collagen has been replaced with autologous connective tissue; (Bi) histological image of ArteFill ${ }^{\circledR}$ at month 3: capillaries are present in the implant and have been integrated into the patient's body ( $\times 40)$; (Bii) histological image taken 10 years after implantation of Artecoll ${ }^{2}$ : connective tissues are mature, characterized by active fibroblasts, microsphere encapsulation, ingrown capillaries, and an absence of immune reaction $(\times 40)$. Image modified with permission. ${ }^{|35|}$

The formulation was later improved with the restriction of microsphere size to $30-50 \mu \mathrm{m}$ to avoid phagocytosis, which in turn decreased the rate of granuloma formation. ${ }^{|36-38|}$ Either bovine collagen (Artecoll ${ }^{\circledR}$ or Artefill $®$; Artes Medical, San Diego, CA, USA) or HA (Dermalive ${ }^{\circledR}$; Dermatech, Paris, France) can be used to suspend PMMA microspheres in order to facilitate the deposition of collagen in the surrounding region. It is important to note that unlike other injectable fillers, Artefill ${ }^{\circledR}$ stimulates the patient's own collagen while keeping the microspheres well-dispersed secondary to their viscosity [Figure 3A]. ${ }^{[35]}$ When the collagen is resorbed, the microspheres persist and become encapsulated by connective tissue, resulting in a bulking effect. ${ }^{[35]}$ As demonstrated in Figure 3B, fibroblasts and collagen fibers have completely enveloped the PMMA microspheres three months following injection with Artefill ${ }^{\circledR}$ [Figure 3B]. ${ }^{[35]}$ Allergy testing is required prior to use since the collagen contained in the product is of bovine origin.

Cohen and Holmes ${ }^{139]}$ compared 123 patients treated with bovine collagen with 128 patients treated with $\operatorname{Artecoll} \circledast$ for the treatment of 1,334 wrinkles (nasolabial folds, radial upper lip lines, glabella lines, and corner of mouth lines). One month after injection, only glabellar lines showed noticeable improvement, and collagen achieved better results. By month 3 , the nasolabial folds and corner of the mouth lines treated with Artecoll ${ }^{\circledR}$ also showed significant improvements. Six months following injection, Artecoll ${ }^{\circledR}$ demonstrated a superior improvement in nasolabial folds when compared to collagen and also had fewer adverse effects, although these differences were not statistically significant. In another study in which 950 patients were surveyed about their experience with Artecoll ${ }^{\circledR}$ for the long lasting correction of wrinkles, 515 surveys were returned. $29 \%$ of the respondents felt that the treatment was very good, $38 \%$ felt that it was good, $23 \%$ found it satisfactory, and $8 \%$ reported no difference. $91 \%$ of the respondents indicated their willingness to repeat the treatment. ${ }^{[40]}$ Carvalho Costa et al. ${ }^{[41]}$ carried out a study in which PMMA injections were administered to 266 patients (154 women, age: 17-72 years), with each patient receiving from 1-4 injections over an interval of 40 to 60 days. The average number of sessions required was four, with $8 \mathrm{~mL}$ of PMMA used per session. While $90 \%$ of the patients reported satisfactory results while the remaining $10 \%$ reported mild improvements, $20 \%$ of the patients experienced temporary adverse effects including swelling, bruising and erythema with no late complications. ${ }^{[41]}$

Overall, PMMA-based fillers are inexpensive, readily accessible and simple to use. Although immediate adverse reactions have been reported in many of the studies, these primarily consist of lumps or nodules which may appear immediately following injection and in some cases several years later.

\section{Poly-L-lactic acid}

As a biodegradable thermoplastic polymer, PLA is extracted 
from renewable sources and has been commercially available as Sculptra/Fill ${ }^{\circledR}$ (Sanofi Aventis, Paris, France). It is injected into either the deep dermis or subcutaneous layer and immediately fills the space, restoring facial fat loss. ${ }^{[42]}$ Once injected, the material creates immediate volumization secondary to the properties associated with the product's microparticles. ${ }^{[43,44]}$ However, the volumization effect disappears within a few months as the microparticles degrade and the material is metabolized, resulting in dermal fibroplasia. ${ }^{[43]}$ This degradation results in the formation of connective tissue or neocollagenesis. ${ }^{[43,45]}$ It is important to understand that PLA is a bio-stimulatory agent which benefits from the host system. ${ }^{[46]}$ The results associated with this product therefore are not immediate and are instead gradual, lasting for at least 2 years. ${ }^{[45,47]}$ It is approved in Europe for the treatment of scars and wrinkles, and in the United States for the treatment of facial lipoatrophy in patients diagnosed with human immunodeficiency virus (HIV) lipodystrophy syndrome. The injection technique is of crucial importance given the risk of papule and nodule formation. ${ }^{[43,45,48]}$ This product is overall very advantageous because it provides volume restoration while simultaneously increasing the dermal thickness. ${ }^{[45,47]}$ Other advantages include reduced safety concerns because it provides temporary results, and its very good risk-benefit profile in both HIV + and cosmetic patients..$^{[42]}$

PLA was first approved for use in Europe in 1999. Subsequently, many studies have been performed to determine the best method for use and the potential benefits. A preclinical study was conducted in 1993 by Gogolewski et al. ${ }^{[44]}$ in which PLA solids were implanted into mice. There were no signs of abscess formation, acute inflammatory response, or cytotoxic effects at the site of implantation. Another study was conducted with 300 patients who underwent treatment with PLA between 1999 and 2004. In total, 819 injections were administered for treatment of the nasolabial folds, labiomental creases, temples, upper lip, cheeks, chin, and marionette lines. Improvements were noted in most patients with effects lasting for $12-24$ months. ${ }^{[49]}$ Ten percent of patients who received treatments between 1999 and 2002 reported subcutaneous papules, which lasted for 12-24 months and resolved without further intervention. Papules can also be treated with intralesional triamcinolone $(10 \mathrm{mg} / \mathrm{mL})$ and/or intralesional 5-fluorouracil with resolution within 3 months. Since 2002, the reported rate of granuloma formation is less than $1 \%$. This low rate is attributed to pre-treatment of PLA with $3 \mathrm{~mL}$ of sterile water $36-48 \mathrm{~h}$ prior to treatment, followed by adding $2 \mathrm{~mL}$ of $1 \%$ lidocaine immediately prior to injection. This creates a dilution of $5 \mathrm{~mL}$, whereas previously $3 \mathrm{~mL}$ of sterile water only was added 2-12 h prior to use. Injection was administered into subcutaneous fat instead of the deep dermis.

A study was conducted by Valantin et al. ${ }^{[50]}$ in 2003 evaluating 50 patients who received four sets at day 0 and $2 \mathrm{~mL}, 4 \mathrm{~mL}$, $6 \mathrm{~mL}$, maximum $4 \mathrm{~mL}$ of PLA per cheek, monitored for 96 weeks with valuations at 6 weeks, 24 weeks, 48 weeks, 72 weeks and 96 weeks. Substantial improvement of at least $40 \%$ in cutaneous thickness from baseline was demonstrated in over $40 \%$ of patients. Total cutaneous thickness at the onset of the trial was $2.9 \mathrm{~mm}$ without the presence of underlying facial fat. The increases in Cutaneous thickness increased at weeks $6,24,48,72$ and 96 to $5.1,6.4,7.2$, 7.2 and $6.8 \mathrm{~mm}$, respectively. Minimal and localized edema was observed in most patients but resolved spontaneously within $48 \mathrm{~h}$. Fifteen out of fifty patients developed minimal ecchymosis which resolved within 3 days. ${ }^{|50|}$ Patients were compliant with their injections despite the side effects; the feasibility of using PLA for facial lipoatrophy treatment was clearly demonstrated. Moyle et al. ${ }^{[51]}$ conducted an open label study to evaluate the effect of immediate vs. delayed PLA injections for 24 weeks. Patients in the immediate treatment group received PLA treatments on day 1 , as well as 2 and 4 weeks after the initial treatment. Patients in the delayed treatment group received PLA injections at weeks 12,14 , and $16 .^{[51]}$ The study was limited to three injections per side and found similar improvements in 24 patients by both subjective assessment and ultrasound. ${ }^{[51]}$ Interestingly, by week 12 , patients in the immediate treatment group scored significantly higher on the visual analogue scale and had lower levels of anxiety than their delayed treatment counterparts. ${ }^{[51]}$ Overall, it was concluded that PLA injection remained efficacious for more than 18 weeks. ${ }^{[51]}$

Although many studies have examined the safety of PLA, its use is continually refined. ${ }^{[46-48,51]}$ While the above studies investigated the optimal treatment times and methods of application, additional studies have been conducted regarding injection in the periorbital and perioral regions, ${ }^{[52]}$ the use of conservative injection volumes ${ }^{[33]}$, and appropriate dilution volumes. ${ }^{[53,54]}$

\section{Calcium hydroxylapatite}

CaHA and its derivatives are naturally found in human bone and dental enamel. ${ }^{[55]}$ Due to the structural similarity of the CaHA to the mineral portion of bone and its composition of calcium and phosphate ions, it is very biocompatible and therefore does not require skin testing. Radiesse ${ }^{\circledR}$ (Bioform Medical, Inc., San Mateo, CA, USA) consists of CaHA microspheres suspended in an aqueous carboxymethylcellulose gel carrier and is primarily used for the correction of moderate to severe wrinkles and folds and volumization of facial soft-tissue. ${ }^{[1]}$ It is also used for correction of facial lipoatrophy secondary to the human immunodeficiency virus. ${ }^{[1]}$ The microspheres present in Radiesse ${ }^{\circledR}$ are thought to act as a scaffold for new collagen formation. CaHA microspheres (25-45 $\mu \mathrm{m}$ in diameter) are suspended in a carboxymethylcellulose gel carrier in a ratio of $30 \%$ microspheres to $70 \%$ gel by volume. ${ }^{[56]}$ The gel base helps to evenly distribute the microspheres at the injection site, providing immediate volume restoration. ${ }^{[56]}$ After the gel degrades in 2-3 months, the microspheres promote the formation of new tissue formation by collagen deposition. ${ }^{[57]}$ Results typically last for $12-18$ months $^{[58-60]}$ leaving behind calcium and phosphate ions after undergoing the phagocytosis process by the macrophages. ${ }^{[57]}$ A study conducted by Marmur et al. ${ }^{[57]}$ evaluated the histologic and electron microscopic structural changes observed following CaHA injection at 1 and 6 months. Standard light microscopy imaging of tissue specimens at 1 month post-injection demonstrated no inflammatory cell response or fibrosis, 
whereas light microscopy sections at 6 months post-injection showed tight aggregates of microspherules surrounded by thick collagen and histiocytes. Multinucleated giant cells surrounding each microspherule were also observed. All treated subjects reported high satisfaction levels at 6 months, and there were no reports of adverse reactions.

Another pivotal study testing the efficacy of Radiesse ${ }^{\circledR}$ for the treatment of nasolabial folds involved 117 subjects with a random split-face design. Radiesse ${ }^{\circledR}$ was injected on one side and collagen was injected on the other. ${ }^{[61]}$ Radiesse ${ }^{\circledR}$ was found to be superior to collagen in $79 \%$ of the folds after 6 months, and required almost half of the volume that was used with collagen. ${ }^{[61]}$ The long-term safety and efficacy of Radiesse ${ }^{\circledR}$ was also tested in the treatment of nasolabial folds by extending the previous study and offering retreatment between 6 and 12 months. ${ }^{[60]}$ Out of a total of 117 subjects, 102 patients enrolled and were evaluated at 39 months after the last injection. ${ }^{[60]}$ At 30 months following the last treatment with Radiesse ${ }^{\circledR}$, improvement was still observed in $40 \%$ of the folds, and no adverse events were reported. ${ }^{[60]}$ Safety and efficacy has also been tested for an injectable implant in which where researchers at two different treatment centers injected CaHA into 113 subjects..$^{|5|}$ 75 patients received single injections and 38 had multiple sessions, with most patients receiving $1 \mathrm{~mL}$ per session. ${ }^{[5]}$ The results suggested a very favorable safety profile with a very high patient satisfaction rating (over $90 \%$ indicated that their results were "good" or "very good") and only 7 minor adverse events which resolved within one month. ${ }^{[5]}$

Although the majority of studies did not show any adverse effects, side effects of Radiesse ${ }^{\circledR}$ may include edema, erythema and transient lumpiness. ${ }^{|63|}$ Radiesse ${ }^{\circledR}$ is not recommended for lip injection because of the frequent occurrence of mucosal nodules. ${ }^{[58]}$ Radiesse ${ }^{\circledR}$ may not be suitable for the treatments of periorbital and glabellar rhytids secondary to safety concerns regarding embolism and necrosis. A case was reported in which a 35-year-old women experienced nausea, vomiting, headache, ptosis and periorbital pain ten minutes following CaHA injection into the nasal tip and dorsum. The symptoms worsened and resulted in sudden monocular vision loss. ${ }^{[62]}$ Although many of the fillers discussed may be associated with very mild temporary reactions, the injector must remain alert to the risk of more serious adverse reactions.

\section{Permanent fillers}

Silicone

A primary consideration in the choice of filler is the degree of maintenance that is required. Some advantages associated with permanent fillers include low maintenance as a regular schedule for re-treatmentis not required, and a lower overall cost because the procedure is performed only once.

Liquid injectable silicone, commonly available as Silikon 1000 and AdatoSil 5000, is classified as a permanent filler for soft tissue augmentation. The chemical composition of the material is predominantly dimethyl polysiloxane, which is available as fluid with variable levels of viscosity. It is injected into either the deep dermis or subcutaneous fat with the aim of inducing 1 fibroplasia and volumization. ${ }^{\text {[63] }}$ Moreover, it is characterized as a clear, colorless fluid with general physiological inertness and resistance to decomposition under extreme temperatures. ${ }^{[63]}$ If the product is inadvertently injected into the blood stream, it behaves very much like injections of air; small doses of the material are distributed throughout the body, while large doses can cause various severe complications including emboli and cellulitis. ${ }^{[63]}$ This product is overall very advantageous in the correction of facial defects, and other intradermal treatments that previously required excessive surgical intervention. ${ }^{[63]}$

Injectable silicone oils for augmentation of the facial tissues were approved by the FDA in the early 1960 s. $^{[64]}$ In spite of the tremendous criticism that silicone has received due to its adverse complications, it has been shown to be very effective in facial augmentation. ${ }^{[64]}$ A clinical study conducted by Hevia ${ }^{[65]}$ demonstrated the efficacy of silicone oil in soft tissue augmentation. In total 916 patients were treated with PDMS-1000 (purified polydimethylsiloxane), an FDA approved silicone oil, over a six year period during which only $1 \%$ showed adverse granulomatous reactions. ${ }^{[65]}$ All treatments were performed with a serial puncture technique, which is well-suited for the injection of silicone oil. ${ }^{[65]}$ Narins and Beer ${ }^{[64]}$ note that the oil must be injected into the immediate subdermal plane or deeper to avoid dermal erythema and ridging. The volume should be limited to no more than $0.5 \mathrm{~mL}$ for small areas including the nasolabial fold, and $2.0 \mathrm{~mL}$ for larger areas as seen in facial lipoatrophy. ${ }^{[64]}$

Another study by Jones et al. ${ }^{[66]}$ evaluated the injection of liquid silicone into HIV patients with facial lipoatrophy. In this study, 77 patients received either Silikon ${ }^{\circledR} 1000$ (Alcon Inc., Fort Worth, TX, USA) or VitreSil ${ }^{\circledR} 1000$ (Richard James Inc., Peabody, MA, USA). Less than $2 \mathrm{~mL}$ of silicone oil was repeatedly injected at intervals of at least a month until a state of "prelipoatrophy" was achieved. Most patients developed erythema and edema following injection which resolved within 3 days. Patient satisfaction in this study was reported to be high although specific details were not provided.

Although many studies have shown the efficacy of silicone oil, an equal number of studies have highlighted adverse reactions secondary to misuse. ${ }^{|9|}$ The improper use of silicone oils including incorrect dosage, improper technique, and impurity of materials has resulted in serious repercussions including cellulitis, product migration, and death. ${ }^{[9]}$ A report by Requena et al. ${ }^{[67]}$ presents 4 cases in which patients experienced moderate to severe complications following silicone injections.

All 4 patients were diagnosed with orofacial granulomatosis after 8-12 months of treatment. ${ }^{[56]}$ It was suggested that the histological appearance of the odemas be studied as they give direct information regarding the form and purity of silicone used, including solid elastomer silicone and oil/ gel silicone. ${ }^{[56]}$ The proper use of liquid silicone, i.e. small volumes, high purity, and the microdroplet technique, is 
believed to have a rate of complications of less than $1 \%{ }^{[67]}$ Granulomas may develop weeks or even years following treatment, and can be managed using oral or intralesional corticosteroids and antibiotics.

The extent to which volume loss and fat atrophy affect facial aging is evident by the large variety of existing intradermal fillers. Intradermal fillers are not as strictly regulated as prescription drugs, as most fillers fall under the category of a medical device rather than that of a pharmaceutical agent. While intradermal fillers are considered to be inert, many of them elicit immunogenic reactions and granulomas. In addition, some fillers induce neo-collagenesis. Temporary fillers generally persist for 3-6 months. While permanent fillers may be advantageous in terms of their longevity, a permanent dermatological procedure may eventually become unnatural as it is unable to accommodate the patient's aesthetic needs over time due to the dynamic nature of facial aging.

\section{Other fillers}

Apart from the fillers mentioned above, a combination of two or more fillers have been shown to be very effective in facial volumization. A 6-month comparison study examined the efficacy and safety profiles of plain CaHA and CaHA mixed with lidocaine for the treatment of nasolabial folds. ${ }^{[68]}$ In this study, 16 patients with moderate-to-deep wrinkle ratings were recruited. A visual analog pain scale was used to assess the patients' pain perception. A blinded injector and an independent observer determined the efficacy of treatment over a period of 24 weeks. There were no significant adverse events and very few local adverse events. Both of the groups reported satisfaction with the treatment and much less pain when CaHA was mixed with lidocaine as compared to plain CaHA. ${ }^{[68]}$ Intradermal fillers have also been used with other anti-aging chemicals including BOTOX ${ }^{\circledR}$. A study by Coleman and Carruthers ${ }^{[69]}$ showed that in younger subjects with glabellar frown lines, treatment with BOTOX ${ }^{\circledR}$ - alone did not show positive results, but when combined with intradermal fillers such as HA gave an immediate resting result and made it last twice as long. Similarly, melomental folds in the perioral region can be effectively treated with the combination of BOTOX ${ }^{\circledR}$ to remove the muscular depressor action of the lower face and intradermal filler to volumize the mouth corners, giving an overall youthful appearance. ${ }^{[69]}$

\section{CONCLUSION}

The field of cosmetic dermatology has been expanding globally. With the ever increasing popularity of nonsurgical enhancement and an increasing number of patients who have been treated, the demand for fillers continues to rise. Research articles, long-term clinical experience, peer reviewed publications and regulatory approvals have all demonstrated the safety and efficacy of various fillers. Fillers appeal to various age demographics including young adults for the enhancement of youthful features, middle-aged adults for early prevention and volume restoration, and the older individuals for delay and maintenance of age-related symptoms. All of the injectable fillers discussed above have their own advantages and disadvantages, and generally do not have any adverse reactions. The optimal filler is determined by the case, cost associated with treatment, and the physician's experience. Research continues to be conducted on formulations, clinical trials, and comparative studies between fillers. Optimal results in treating dynamic lines and volume loss are achieved with combination therapy. It is crucial that patients have realistic expectations as multiple treatments may be required, and each filler has its limitations. More comparative studies and literature reviews are required to provide the layperson with a summary of the many options and their risks and benefits, thereby allowing patients to choose the filler most suitable to their needs.

\section{Financial support and sponsorship}

The authors would like to thank the Grant for Excellent Young Scholars from Xi'an Jiaotong University.

\section{Conflicts of interest}

There are no conflicts of interest.

\section{REFERENCES}

I. Luebberding S, Alexiades-Armenakas M. Facial volume augmentation in 2014: overview of different filler options.J Drugs Dermatol 20।3; 12:1339-44.

2. Maddern G.The Australian Safety and Efficacy Register of New Interventional Procedures - Surgical (ASERNIP-S) assesses new surgeries. Aust Health Rev 2004;28:143-4.

3. Silvipriya KS, Kumar KK, Bhat AR, Kumar BD, John A, Lakshmanan P. Collagen: animal sources and biomedical application. J App Pharm Sci 2015;5:I23-7.

4. Ottani V, Raspanti M, Ruggeri A. Collagen structure and functional implications. Micron 200 I;32:25I-60.

5. Sadick NS, Katz BE, Roy D. A multicenter, 47-month study of safety and efficacy of calcium hydroxylapatite for soft tissue augmentation of nasolabial folds and other areas of the face. Dermatol Surg 2007;33:SI22-6; discussion SI26-7.

6. Baumann L, Kaufman J, Saghari S. Collagen fillers. Dermatol Ther 2006;19:13440.

7. Nicolle FV. Correction of age- and disease-related contour deficiencies of the face. Aesthetic Plastic Surg 1985;9:159-62.

8. Bailin MD, Bailin PL. Correction of surgical scars, acne scars, and rhytids with zyderm and zyplast implants.J Dermatol Surg Oncol 1988; |4:31-3.

9. Zeide DA.Adverse reactions to collagen implants. Clin Dermatol 1986;4:17682.

10. Struck H. Immunological investigations of antigenicity and specificity of soluble collagen fractions. IV. Anaphylaxis and allergy experiments. Eur Surg Res 1976;8:243-9.

II. Monstrey SJ, Pitaru S, Hamdi M,Van Landuyt K, Blondeel P, Shiri J, Goldlust A, Shoshani D. A two-stage phase I trial of Evolence30 collagen for soft-tissue contour correction. Plast Reconstr Surg 2007; 1 20:303-I I.

12. Narins RS, Brandt FS, Lorenc ZP, Maas CS, Monheit GD, Smith SR, Mclntyre S. A randomized, multicenter study of the safety and efficacy of Dermicol-P35 and non-animal-stabilized hyaluronic acid gel for the correction of nasolabial folds. Dermatol Surg 2007;33:S2 I 3-2I; discussion S22I.

13. Lee JH, Choi YS, Kim SM, Kim YJ, Rhie JW, Jun YJ. Efficacy and safety of porcine collagen filler for nasolabial fold correction in Asians: a prospective multicenter, 12 months follow-up study.J Korean Med Sci 20I4;29:S2I 7-2I.

14. Brongo S, Moccia LS, Nunziata V, D'Andrea F. Keratoacanthoma arising after site injection infection of cosmetic collagen filler. Int J Surg Case Rep 20I3;4:429-3I.

15. Tumerdem B, Aydin A, Kesim SN. An unusual case of self-injected bovine fat in liquid form to the face for cosmetic reasons. Aesthetic Plastic Surg 200ss4;28:25I-3.

16. Baumann LS, Shamban AT, Lupo MP, Monheit GD, Thomas JA, Murphy DK, Walker PS; JUVEDERM vs. ZYPLAST Nasolabial Fold Study Group. Comparison of smooth-gel hyaluronic acid dermal fillers with cross-linked bovine collagen: a multicenter, double-masked, randomized, within-subject study. Dermatol Surgery 2007;33:S I28-35.

17. Kim BS, Choi JS, Kim JD, Yoon HI, Choi YC, Cho YW. Human collagen isolated from adipose tissue. Biotechnol Prog 20I2;28:973-80. 
18. Báez J, Olsen D, Polarek JW. Recombinant microbial systems for the production of human collagen and gelatin. Appl Microbiol Biotechnol 2005;69:245-52.

19. Raines RT. Stronger and (now) Longer Synthetic Collagen. Adv Exp Med Biol 2009;61 I:xci-xcviii.

20. Bauman L. CosmoDerm/CosmoPlast (human bioengineered collagen) for the aging face. Facial Plastic Surg 2004;20:125-8.

21. Sclafani AP, Romo T 3rd. Collagen, human collagen, and fat: the search for a three-dimensional soft tissue filler. Facial Plastic Surg 2001; 17:79-85.

22. Stolman LP. Human collagen reactions. Dermatol Surg 2005;3 I:I634.

23. Sclafani AP, Romo T 3rd, Jacono AA. Rejuvenation of the aging lip with an injectable acellular dermal graft (Cymetra). Arch Facial Plast Surg 2002;4:2527.

24. Lowe NJ, Maxwell CA, Lowe P, Duick MG, Shah K. Hyaluronic acid skin fillers: adverse reactions and skin testing. J Am Acad Dermatol 200 I;45:930-3.

25. Narins RS, Brandt F, Leyden J, Lorenc ZP, Rubin M, Smith S. A randomized, double-blind, multicenter comparison of the efficacy and tolerability of Restylane versus Zyplast for the correction of nasolabial folds. Dermatol Surg 2003;29:588-95.

26. Lindqvist C, Tveten S, Bondevik BE, Fagrell D.A randomized, evaluator-blind, multicenter comparison of the efficacy and tolerability of Perlane versus Zyplast in the correction of nasolabial folds. Plast Reconstr Surg 2005; I I 5:282 9.

27. Wang F, Garza LA, Kang S, Varani J, Orringer JS, Fisher GJ, Voorhees JJ. In vivo stimulation of de novo collagen production caused by cross-linked hyaluronic acid dermal filler injections in photodamaged human skin. Arch Dermatol 2007; | 143:155-63.

28. Monheit GD, Coleman KM. Hyaluronic acid fillers. DermatolTher 2006; 19:141 50.

29. Beer K.A randomized, evaluator-blinded comparison of efficacy of hyaluronic acid gel and avian-sourced hylan B plus gel for correction of nasolabial folds. Dermatol Surg 2007;33:928-36.

30. Prager W, Wissmueller E, Havermann I, Bee EK, Howell DJ, Zschocke I, Simon J. A prospective, split-face, randomized, comparative study of safety and I 2-month longevity of three formulations of hyaluronic acid dermal filler for treatment of nasolabial folds. Dermatol Surg 20I 2;38: I I 43-50.

3I. Kim H, Park KY, Choi SY, Koh HJ, Park SY, Park WS, Bae IH, Kim BJ.The efficacy, longevity, and safety of combined radiofrequency treatment and hyaluronic Acid filler for skin rejuvenation. Ann Dermatol 2014;26:447-56.

32. Choi SY, Lee YH, Kim H, Koh HJ, Park SY, Park WS, Bae IH, Park KY, Kim BJ. A combination trial of intradermal radiofrequency and hyaluronic acid filler for the treatment of nasolabial fold wrinkles: a pilot study.J Cosmet LaserTher 20|4;16:37-42.

33. Kanchwala SK, Holloway L, Bucky LP. Reliable soft tissue augmentation. Ann. Plas. Surg. 2005;55:30-5

34. Lemperle G, Ott H, Charrier U, Hecker J, Lemperle M. PMMA microspheres for intradermal implantation: Part I. Animal research. Ann Plast Surg 1991;26:57-63.

35. Vent J, Lemperle G. Prevention and treatment of complications after polymethylmethacrylate-microspheres injections. Facial Plast Surg 20|4;30:628-34

36. Lemperle G, Knapp TR, Sadick NS, Lemperle SM. ArteFill permanent injectable for soft tissue augmentation: I. Mechanism of action and injection techniques. Aesthetic Plastic Surg 2010;34:264-72.

37. Lemperle G, Sadick NS, Knapp TR, Lemperle SM. ArteFill permanent injectable for soft tissue augmentation: II. Indications and applications. Aesthetic Plastic Surg 2010;34:273-86

38. Cohen SR, Berner CF, Busso M, Clopton P, Hamilton D, Romano JJ, et al. Five-year safety and efficacy of a novel polymethylmethacrylate aesthetic soft tissue filler for the correction of nasolabial folds. Dermatol Surg 2007;33:S222-30.

39. Cohen SR, Holmes RE. Artecoll: a long-lasting injectable wrinkle filler material: Report of a controlled, randomized, multicenter clinical trial of 251 subjects. Plast Reconstr Surg 2004; I 14:964-76; discussion 977-9.

40. Lemperle G, Gauthier-Hazan N, Lemperle M. PMMA-Microspheres (Artecoll) for long-lasting correction of wrinkles: refinements and statistical results. Aesthetic Plastic Surg 1998;22:356-65

4I. Carvalho Costa IM, Salaro CP, Costa MC. Polymethylmethacrylate facial implant: a successful personal experience in Brazil for more than 9 years. Dermatol Surg 2009;35:1221-7.

42. Donofrio L, Weinkle S. The third dimension in facial rejuvenation: a review. J Cosmet Dermatol 2006;5:277-83.

43. Bartus C, William Hanke C, Daro-Kaftan E. A decade of experience with injectable poly-L-lactic acid: a focus on safety. Dermatol Surg 20I3;39:698-705.

44. Gogolewski S, Jovanovic M, Perren SM, Dillon JG, Hughes MK. Tissue response and in vivo degradation of selected polyhydroxyacids: polylactides (PLA), poly(3-hydroxybutyrate) (PHB), and poly(3-hydroxybutyrate-co-3hydroxyvalerate) (PHB/VA).J Biomed Mater Res 1993;27:I I35-48.

45. Vleggaar D, Bauer U. Facial enhancement and the European experience with Sculptra (poly-l-lactic acid).J Drugs Dermatol 2004;3:542-7.

46. Fitzgerald $R$,Vleggaar $D$. Facial volume restoration of the aging face with polyI-lactic acid. Dermatol Ther 20 I I;24:2-27.

47. Burgess CM, Quiroga RM. Assessment of the safety and efficacy of poly-Llactic acid for the treatment of HIV-associated facial lipoatrophy. J Am Acad Dermatol 2005;52:233-9.

48. Schierle CF, Casas LA. Nonsurgical rejuvenation of the aging face with injectable poly-L-lactic acid for restoration of soft tissue volume. Aesthet Surg J 201 1;31:95-109.

49. Woerle B, Hanke CW, Sattler G. Poly-L-lactic acid: a temporary filler for soft tissue augmentation. J Drugs Dermatol 2004;3:385-9.

50. Valantin MA, Aubron-Olivier C, Ghosn J, Laglenne E, Pauchard M, Schoen H, Bousquet R, Katz P, Costagliola D, Katlama C. Polylactic acid implants (NewFill) to correct facial lipoatrophy in HIV-infected patients: results of the openlabel study VEGA. AIDS 2003; 1 7:247I-7.

5I. Moyle GJ, Lysakova L, Brown S, Sibtain N, Healy J, Priest C, Mandalia S, Barton SE. A randomized open-label study of immediate versus delayed polylactic acid injections for the cosmetic management of facial lipoatrophy in persons with HIV infection. HIV Med 2004;5:82-7.

52. Lowe NJ, Maxwell CA, Lowe P, Shah A, Patnaik R. Injectable poly-l-lactic acid: 3 years of aesthetic experience. Dermatol Surg 2009;35:344-9.

53. Palm MD, Woodhall KE, Butterwick KJ, Goldman MP. Cosmetic use of poly-Ilactic acid: a retrospective study of I30 patients. Dermatol Surg 2010;36:16170.

54. Rossner F, Rossner M, Hartmann V, Erdmann R, Wiest LG, Rzany B. Decrease of reported adverse events to injectable polylactic acid after recommending an increased dilution: 8-year results from the Injectable Filler Safety study.J Cosmet Dermatol 2009;8:14-8.

55. Hobar PC, Pantaloni M, Byrd HS. Porous hydroxyapatite granules for alloplastic enhancement of the facial region. Clin Plast Surg 2000;27:557-69.

56. Pavicic T. Calcium hydroxylapatite filler: an overview of safety and tolerability. J Drugs Dermatol 2013;12:996-1002.

57. Marmur ES, Phelps R, Goldberg DJ. Clinical, histologic and electron microscopic findings after injection of a calcium hydroxylapatite filler.J Cosmet LaserTher 2004;6:223-6

58. Moers-Carpi M,Vogt S, Santos BM, Planas J,Vallve SR, Howell DJ.A multicenter, randomized trial comparing calcium hydroxylapatite to two hyaluronic acids for treatment of nasolabial folds. Dermatol Surg 2007;33:SI 44-5I.

59. Jacovella PF.Use of calcium hydroxylapatite (Radiesse) for facial augmentation. Clin Interv Aging 2008;3:161-74.

60. Bass LS, Smith SL, Busso M, McClaren M. Calcium hydroxylapatite (Radiesse) for treatment of nasolabial folds: long-term safety and efficacy results. Aesthet Surg J 2010;30:235-8.

6I. Smith S, Busso M, McClaren M, Bass LS.A randomized, bilateral, prospective comparison of calcium hydroxylapatite microspheres versus human-based collagen for the correction of nasolabial folds. Dermatol Surg 2007;33:SI I221; discussion S2I.

62. Chou CC, Chen HH, Tsai YY, Li YL, Lin HJ. Choroid vascular occlusion and ischemic optic neuropathy after facial calcium hydroxyapatite injection- a case report. BMC Surg 2015;15:21.

63. Aronsohn RB. Observations on the use of silicone in the face.Arch Otolaryngol 1965;82:191-4.

64. Narins RS, Beer K. Liquid injectable silicone: a review of its history, immunology, technical considerations, complications, and potential. Plast Reconstr Surg 2006; I 18:77S-84S.

65. Hevia O. Six-year experience using 1,000 -centistoke silicone oil in 916 patients for soft-tissue augmentation in a private practice setting. Dermatol Surg 2009;35:1646-52.

66. Jones DH, Carruthers A, Orentreich D, Brody HJ, Lai MY, Azen S, Van Dyke GS. Highly purified 1000-cSt silicone oil for treatment of human immunodeficiency virus-associated facial lipoatrophy: an open pilot trial. Dermatol Surg 2004;30:1279-86.

67. Requena C, Requena L, Alegre V, Serra C, Llombart B, Nagore E, Guillén C, Sanmartín O. Adverse reaction to silicone simulating orofacial granulomatosis. J Eur Acad Dermatol Venereol 2015;29:998-I00I.

68. Narins RS, Baumann L, Brandt FS, Fagien S, Glazer S, Lowe NJ, Monheit GD, Rendon MI, Rohrich RJ,Werschler WP.A randomized study of the efficacy and safety of injectable poly-L-lactic acid versus human-based collagen implant in the treatment of nasolabial fold wrinkles. J Am Acad Dermatol 20 I 0;62:448-62.

69. Coleman KR, Carruthers J. Combination therapy with BOTOX and fillers: the new rejuvnation paradigm. Dermatol Ther 2006;19:177-88. 